\title{
Evaluación del desempeño docente por áreas de conocimiento: El caso de la Facultad de Ciencias Administrativas y Sociales de la Universidad Autónoma de Baja California, México
}

Teaching Evaluation by areas of knowledge: the case of Faculty of Social and Administrative Sciences, University Autonomous of Baja California, Mexico

\section{Volumen 21, Número 3}

Setiembre - Diciembre

pp. $1-20$

Patricio Henríquez Ritchie

Vicente Arámburo Vizcarra 


\title{
Evaluación del desempeño docente por áreas de conocimiento: El caso de la Facultad de Ciencias Administrativas y Sociales de la Universidad Autónoma de Baja California, México
}

Teaching Evaluation by areas of knowledge: the case of Faculty of Social and Administrative Sciences, University Autonomous of Baja California, Mexico

\author{
Patricio Henríquez Ritchie ${ }^{1}$ \\ Vicente Arámburo Vizcarra ${ }^{2}$
}

Resumen. La Facultad de Ciencias Administrativas y Sociales (FCAYS) de la Universidad Autónoma de Baja California (UABC) evalúa a su cuerpo docente desde la opinión del estudiantado todos los semestres. Para ello, considera cuatro dimensiones (planificación, manejo de contenidos, estrategias de enseñanza, evaluación del aprendizaje), con propósitos formativos y sumativos. A partir de tres áreas de conocimiento (ciencias administrativas, sociales y jurídicas), se exponen los resultados cuantitativos de este estudio: análisis descriptivos de las dimensiones evaluadas y análisis inferenciales para contrastar la hipótesis de independencia entre las áreas de conocimiento y la opinión del estudiantado acerca del desempeño de sus docentes. Los datos se recopilan por medio de un cuestionario de opción múltiple que incluye dieciséis variables, aplicado en el primer semestre del 2019. En general, los resultados descriptivos muestran una opinión positiva acerca de la planificación de las clases del profesorado, así como del dominio de contenidos, aunque con algunas diferencias significativas según el área de conocimiento. El estudiantado de Ciencias Sociales opina que sus docentes son más proclives al uso de estrategias apegadas a las teorías psicológicas constructivistas de la enseñanza y el aprendizaje, de ahí que muestren una opinión más positiva acerca de las estratrategias de evaluación. Esto contrasta significativamente con el de Ciencias Jurídicas, el cual opina que sus docentes utilizan estrategias más identificadas con el paradigma conductista, por lo que muestra una opinión más negativa en torno a las estrategias de evaluación del aprendizaje.

Palabras clave: evaluación docente, estrategias de enseñanza, evaluación del aprendizaje.

Abstract. The Faculty of Administrative and Social Sciences (FCAYS) of the Autonomous University of Baja California (UABC) evaluates their teaching body from the opinion of the student body every semester considering four dimensions (planning, content management, teaching strategies, learning evaluation), for formative and summative purposes. From three areas of knowledge (administrative, social and legal sciences), the quantitative results of this study are presented: descriptive analysis of the evaluated dimensions and inferential analyzes to contrast the hypothesis of independence between the areas of knowledge and the opinion of the students. about the performance of their teachers, through a multiple-choice questionnaire that includes 16 variables applied in the first semester of 2019. In general, the descriptive results show a positive opinion about the planning of the teachers' classes, as well as the mastery of content, although with some significant differences according to the area of knowledge. Social Sciences students believe that their teachers are more inclined to use strategies attached to constructivist psychological theories of teaching and learning, showing a more positive opinion about evaluation strategies. This contrasts significantly with those of Legal Sciences, who believe that their teachers use strategies more identified with the behaviorist paradigm, along with showing a more negative opinion about learning assessment strategies.

Keywords: teaching evaluation, teaching strategies, learning strategies.

\footnotetext{
1 Profesor-Investigador de la Universidad Autónoma de Baja California (UABC), Baja California, México. Doctor en Ciencias Educativas por la misma institución. Dirección electrónica: phenriquez@uabc.edu.mx Orcid https://orcid.org/0000-0002-1026-3379

2 Profesor-Investigador de la Universidad Autónoma de Baja California (UABC), Baja California, México. Doctor en Ciencias Educativas por la misma institución. Dirección electrónica: vicente@uabc.edu.mx Orcid https://orcid.org/0000-0002-9673-2438
}

Artículo recibido: 18 de marzo, 2021

Enviado a corrección: 14 de junio, 2021

Aprobado:16 de agosto, 2021 


\section{Introducción}

La evaluación del desempeño docente en el nivel educativo superior ha sido un tema de gran trascendencia durante los últimos años tanto a nivel internacional como nacional y se ha enfocado principalmente en aspectos de planificación de clases, organización de las actividades de enseñanza y dominio de los contenidos por parte del profesorado, debido a que son las dimensiones que se consideran trascendentales dentro de la función docente (Bravo Mancero y Varguillas Carmona, 2015; Contreras y Díaz Quero, 2007; Gómez y Valdés, 2019; Ortiz Fajardo, 2009). Sin embargo, se destaca la necesidad de recurrir a otros sujetos más allá del estudiantado y a otras estrategias evaluativas (autoevaluación, coevaluación), así como de profundizar la comprensión de otras dimensiones, tales como las estrategias didácticas que utiliza el cuerpo docente para impartir los contenidos de sus materias y las estrategias de evaluación del aprendizaje. El presente artículo aborda la segunda de las problemáticas mencionadas anteriormente: a partir de la opinión del estudiantado de la FCAYS de la UABC, período 2019-1, se indagan las estrategias de enseñanza que utilizan sus docentes, así como las estrategias de evaluación del aprendizaje que implementan en torno a los contenidos enseñados. Con base en los resultados expuestos, se pretenden trazar los lineamientos necesarios para promover una de las funciones trascendentales de la evaluación educativa, a saber (Jornet y Leyva, 2009): la retroalimentación continua, el seguimiento y el mejoramiento procesual y sistemático de la realidad educativa evaluada.

La función formativa de la evaluación del desempeño docente exige que los resultados aportados por los estudios al repecto vayan más allá de la rendición final de cuentas y el otorgamiento de estímulos salariales con base en dichos resultados (Gómez y Valdés, 2019). En este sentido, es necesario superar los modelos evaluativos centrados en la acreditación laboral, la promoción profesional o los incrementos salariales para el personal docente (UNESCO, 2006). También se destaca la necesidad de promover procesos de autoevaluación y coevaluación docente, de manera que el estudiantado no sea el único agente evaluador del desempeño de su profesorado. El presente documento pretende profundizar la comprensión de algunas dimensiones del desempeño docente que ayuden a retroalimentar y a mejorar aspectos didácticos, relacionados principalmente con estrategias de enseñanza y evaluación del aprendizaje. Lo anterior se realiza a partir del análisis de la relación entre dichas dimensiones y las áreas de conocimiento en las que se desenvuelve el estudiantado y el cuerpo docente de la FCAYS de la UABC. 
Los objetivos de este artículo son: i) describir la opinión del estudiantado de la FCAYS de la UABC acerca del desempeño de sus docentes y ii) analizar la relación entre las áreas de conocimiento y la opinión del estudiantado de la FCAYS de la UABC acerca del desempeño de sus docentes. A continuación, se expone un análisis teórico en función de estos dos objetivos de investigación.

\section{Referentes teóricos}

El debate teórico en torno a lo que significa ser una persona docente efectiva lleva varios años de desarrollo. Al respecto, la literatura ha dado cuenta claramente de dos posturas diferentes: por un lado, la investigación sobre la enseñanza, realizada a partir de la década de 1990 con estudios como los de Schoenfeld (1998), predominantemente en el mundo anglosajón y orientada a construir modelos para definir las características de un buen profesorado eficaz; por el otro, las posturas de corte constructivista, planteadas por Coll y Solé (2002), que sostienen que es prácticamente imposible encontrar un modelo general al respecto.

Para algunos autores (Espinoza-Freire, Tinoco-Izquierdo y Sánchez-Barreto, 2017; Hativa, 2000; Santiago-García y Fonseca-Bautista, 2016), un buen o buena docente es quien logra que sus estudiantes obtengan un aprendizaje profundo y significativo. Por su parte, Hativa, Barak y Simhi (2001) identificaron una serie de atributos relacionados con el logro estudiantil: a) preparación de las clases y organización del maestro; b) claridad en el manejo de los contenidos; c) capacidad para estimular el interés del estudiantado y propiciar la motivación para el estudio, a través la manifestación de expresiones de entusiasmo; d) capacidad de propiciar relaciones positivas con los alumnos; e) mantención de altas expectativas de logro; y f) establecimiento de un clima positivo dentro del salón de clases.

Por su parte, algunas vertientes dentro del paradigma constructivista parten de la premisa de que la enseñanza es un proceso esencialmente socio-cultural (Coll y Solé, 2002). En este sentido, lo importante no es la búsqueda de los mecanismos y de los procedimientos mediante los cuales la eficacia docente influye en el proceso educativo. El énfasis se debe centrar en la interactividad y en la construcción progresiva de significados compartidos entre el profesorado y el estudiantado, así como en el proceso de transferencia progresiva del control de aquel a este. De esta manera, Coll y Solé (2002) proponen como elemento medular un triángulo en el que simultáneamente interactúan los contenidos, el estudiantado y el profesorado. Este enfoque permite destacar la importancia de los contenidos curriculares y las 
características específicas de la disciplina en cuestión, junto con un análisis de las interacciones del profesorado y del alumnado en torno a los contenidos.

De acuerdo con Stake y Cisneros (2000) y Stake (2008), una evaluación efectiva del desempeño docente debe estudiar y considerar las metas institucionales, el ambiente en el salón de clases, la organización y los procedimientos administrativos, los contenidos curriculares, el logro del estudiantado y el impacto de los programas educativos en la sociedad. La enseñanza se puede juzgar adecuadamente solo dentro del contexto en el que interactúan estos factores. Por tanto, dada la complejidad de la docencia, resulta complejo diseñar e instrumentar modelos de evaluación que sistematicen los factores que la explican, la determinan y que permiten su comprensión.

Un problema insoslayable que ha sido destacado por algunos autores respecto a la evaluación del desempeño docente (Abreu, Naranjo, Rhea y Gallegos, 2016; Espinoza-Freire et al., 2017; Hativa, 2000; Santiago-García y Fonseca-Bautista, 2016) es la percepcón del estudiantado al respecto y los sesgos implicados en dicha percepción como única fuente de información para evaluar al profesorado. Se recomienda recurrir a fuentes de información adicionales (directivos, especialistas, colegas) con el fin de lograr una evaluación objetiva, válida, confiable y libre de sesgos. Sin embargo, cuando el interés está puesto en la evaluación de los espectos estratégicos del desempeño docente, la percepción del estudiantado se puede utilizar como un recurso pertinente e idóneo, recuperada a partir de instrumentos válidos y confiables.

En este trabajo se pretende continuar con el interés de otras investigaciones que han intentado explicar, analizar y comprender el uso de estrategias de enseñanza a nivel universitario (Abreu et al., 2016; Ávila Freites, Quintero y Hernández, 2010; Bravo Mancero y Varguillas Carmona, 2015; Contreras y Díaz Quero, 2007; Espinoza Silva, 2009; Ortiz Fajardo, 2009). Particularmente, se exploran dimensiones del desempeño docente asociadas con el manejo y el dominio de los contenidos, las estrategias de enseñanza-aprendizaje utilizadas, así como las formas de evaluación del aprendizaje. Todo lo anterior desde la opinión del estudiantado. A su vez, se exploran eventuales relaciones significativas entre el uso de estrategias de enseñanza y el área de conocimiento en el que enseña el cuerpo docente. De esta manera, se intenta abonar a la discusión sobre la importancia del tipo enseñanza de acuerdo con la naturaleza de la disciplina o campo de conocimiento.

En la UABC se aplica semestralmemte un cuestionario de evaluación docente al estudiantado, el cual debe contestarlo de manera obligatoria. Los resultados de este 
cuestionario funcionan de base para promover cursos de capacitación docente en las áreas con debilidades. Dentro de la FCAYS, se aplica un cuestionario de evaluacion del desempeño docente desde la mirada del estudiantado que busca conocer su opinión acerca de la planificación de clsases, el dominio de contenidos, las estrategias de enseñanza y las estrategias de evaluación del aprendizaje del profesorado. Los análisis previos de la información recogida a través de este cuestionario sugieren que las áreas de conocimiento es un factor determinante en las estrategias de enseñanza y de evaluación del aprendizaje. De aquí el interés de exponerlo en el presente documento.

\section{Metodología}

\subsection{Enfoque}

El presente estudio se reconoce como una investigación cuantitativa, de alcance descriptivo y correlacional, siguiendo los patrones de un diseño observacional, prospectivo y transversal.

\subsection{Unidades de análisis}

La FCAYS de la UABC (México) se compone de ocho licenciaturas que abarcan tres áreas de conocimiento: i) ciencias jurídicas (licenciatura en Derecho); ii) ciencias administrativas (licenciaturas en Administración de Empresas, en Contaduría y en Informática); y iii) ciencias sociales (licenciaturas en Psicología, en Sociología, en Comunicación y en Educación), tal como se observa en la tabla 1. Durante el período 2019-1, las licenciaturas que aportaron mayor representatividad de participantes respecto a su población fueron Educación ( $\mathrm{n}=333$; $86.5 \%)$ y $\operatorname{LAE}(n=531 ; 86.3 \%)$, mientras que la licenciatura en Comunicación aportó la representatividad más baja $(n=470 ; 37.7 \%)$. Por áreas de conocimiento, las licenciaturas englobadas en ciencias sociales (educación, psicología, sociología y comunicación) representaron el mayor porcentaje de participantes ( $n=987 ; 40.7 \%)$, mientras que las ciencias jurídicas (licenciatura en derecho) aportaron la menor cantidad de estudiantes ( $n=571 ; 23.6 \%$ ). 
Tabla 1.

Población, muestra, áreas de conocimiento y etapas que cursan estudiantes, FCAYS, UABC (México), 2019-1

\begin{tabular}{|c|c|c|c|c|c|c|}
\hline FCAYS & $\mathbf{N}$ & n & $\%$ & Áreas de conocimiento & $\mathbf{n}$ & $\%$ \\
\hline Sociología & 95 & 72 & 75.8 & \multirow{4}{*}{ Ciencias Sociales } & \multirow{4}{*}{987} & \multirow{4}{*}{40.7} \\
\hline Psicología & 880 & 405 & 46.0 & & & \\
\hline Educación & 385 & 333 & 86.5 & & & \\
\hline Comunicación & 470 & 177 & 37.7 & & & \\
\hline $\begin{array}{l}\text { Adminis. } \\
\text { Empresas }\end{array}$ & 531 & 458 & 86.3 & \multirow{3}{*}{$\begin{array}{c}\text { Ciencias } \\
\text { Administrativas }\end{array}$} & \multirow{3}{*}{865} & \multirow{3}{*}{35.7} \\
\hline Contaduría & 528 & 295 & 55.9 & & & \\
\hline Informática & 215 & 112 & 52.1 & & & \\
\hline Derecho & 1244 & 571 & 45.9 & \multirow{2}{*}{ Ciencias Jurídicas } & \multirow{2}{*}{571} & \multirow{2}{*}{23.6} \\
\hline TOTAL & 4348 & 2423 & 55.7 & & & \\
\hline
\end{tabular}

Fuente: elaboración propia, 2021

Cabe destacar que la muestra de participantes se seleccionó de manera aleatoria, buscando la mayor representatividad sin regirse bajo algoritmos probabilísticos. Con base en la población estudiantil del período 2019-1, se seleccionaron aleatoriamente salones de clases de todas las licenciaturas de la FCAYS, incluyendo al menos un salón de clases de cada semestre por cada licenciatura. Así, se contó con una muestra aleatotria no probabilística de la percepción de los estudiantes del primer al octavo demestre de cada programa de estudios de la Facultad.

\subsection{Técnicas de recolección de información}

Como se mencionó, en la FCAYS se realiza semestralmente el proceso de evaluación del desempeño docente desde la opinión del estudiantado. En el presente artículo se exponen los principales resultados de este proceso correspondiente al período 2019-1. En el marco de lo anterior, se elaboró y validó un instrumento compuesto por cuatro dimensiones (Henríquez, Arámburo y Dávila, 2017): i) organización y planeación; ii) manejo o dominio de contenidos; iii) estrategias de enseñanza-aprendizaje (procedimientos, técnicas y recursos); y iv) evaluación del aprendizaje (métodos e instrumentos de evaluación). A continuación, se detalla cada dimensión, sus variables y sus escalas de medición (tabla 2): 
Tabla 2.

Dimensiones y variables del cuestionario de evaluación del desempeño docente

\begin{tabular}{|c|l|c|}
\hline \multicolumn{2}{|c|}{ (FCAYS, UABC, México) } \\
\hline \multirow{3}{*}{$\begin{array}{c}\text { Organización y } \\
\text { planeación }\end{array}$} & \multicolumn{1}{|c|}{ VARIABLES } & ESCALA \\
\cline { 2 - 3 } & Entrega y explicación del programa & Nominal \\
\cline { 2 - 3 } & Respecto del programa durante el semestre & Ordinal \\
\cline { 2 - 3 } & Asistencia a clases & Ordinal \\
\hline \multirow{3}{*}{$\begin{array}{c}\text { Manejo y dominio de } \\
\text { contenidos }\end{array}$} & Explicación clara y secuencial de los contenidos & Ordinal \\
\cline { 2 - 3 } & Conexión con situaciones reales y cotidianas & Ordinal \\
\cline { 2 - 3 } & Conexión con otras materias & Ordinal \\
\cline { 2 - 3 } & Fomento de participación en torno a contenidos & Ordinal \\
\cline { 2 - 3 } $\begin{array}{c}\text { Estrategias de } \\
\text { enseñanza-aprendizaje }\end{array}$ & Establecimiento de reglas y normas & Ordinal \\
\cline { 2 - 3 } & Estrategias y técnicas de enseñanza & Nominal \\
\cline { 2 - 3 } & Uso de recursos didáctico de apoyo & Nominal \\
\hline \multirow{3}{*}{$\begin{array}{c}\text { Evaluación del } \\
\text { aprendizaje }\end{array}$} & Oantidad de evaluaciones & De razón \\
\cline { 2 - 3 } & Retroalimentación & Nominal \\
\cline { 2 - 3 } & Instrumentos & Ordinal \\
\cline { 2 - 3 } & Opinión acerca de estrategias de evaluación & Oranal \\
\hline
\end{tabular}

Fuente: elaboración propia, 2021

La aplicación del instrumento se realizó en abril de 2019. El procedimiento implicó la selección aleatoria de distintos semestres por cada licenciatura, priorizando al menos un grupo de cada semestre. Esto permite abarcar las opiniones del estudiantado de todos los semestres por cada programa educativo.

\subsection{Procesamiento de análisis}

El análisis de los datos recolectados se efectuó por medio del paquete estadístico SPSS versión 23.0. Se obtuvieron los indicadores descriptivos básicos (distribuciones de frecuencia, indicadores de tendencia central e índices de dispersión) por cada dimensión y se realizaron pruebas de contraste de independencia de variables (chi-cuadrado, $X^{2}$ ), con niveles de significancia de 0.05 . Lo anterior con el objetivo de analizar la relación entre la variable áreas de conocimiento y las variables relacionadas con las cuatro dimensiones que componen el cuestionario, principalmente las estrategias de enseñanza y de evaluación del aprendizaje. La aplicación de este análisis estadístico no-paramétrico se justificó a partir de las escalas con las que se estimaron las variables (nominal categórica y ordinal). 


\section{Resultados}

En una primera instancia, se preguntó la opinión del estudiantado acerca del manejo de contenidos por parte de sus docentes (tabla 3). Al respecto, se utilizó una escala de Likert de cuatro pasos entre "nunca" y "siempre", la cual se recodificó en dos niveles de frecuencia: baja (nunca, ocasionalmente) y alta (frecuentemente, siempre). A nivel general, se observó una opinión positiva en torno a la capacidad de explicación $(85.6 \%)$ y dominio $(87.0 \%)$ de los contenidos que enseña el personal docente. Asimismo, un porcentaje similar asevera que el cuerpo docente relaciona los contenidos con otras materias y fomenta la participación del estudiantado en sus clases con una frecuencia alta. Sin embargo, llama la atención que cerca de la mitad de participantes $(42.1 \%$ ) constata que nunca o solo ocasionalmente el profesorado contextualiza la enseñanza de los contenidos de sus materias con situaciones, experiencias o problemas relacionados con su vida cotidiana.

Tabla 3.

Manejo de contenidos por parte del cuerpo docentes, FCAYS, UABC (México) 2019-1

\begin{tabular}{|l|c|c|c|c|}
\hline \multirow{2}{*}{} & \multicolumn{2}{|c|}{ Bajo } & \multicolumn{2}{c|}{ Alto } \\
\cline { 2 - 5 } & $\mathbf{n}$ & $\mathbf{\%}$ & $\mathbf{n}$ & \% \\
\hline Explicación & 345 & 14.4 & 2056 & 85.6 \\
\hline Dominio & 312 & 13.0 & 2088 & 87.0 \\
\hline Contextualización & 1004 & 42.1 & 1383 & 57.9 \\
\hline Otras materias & 337 & 14.1 & 2059 & 85.9 \\
\hline Participación & 365 & 15.2 & 2029 & 84.8 \\
\hline
\end{tabular}

Fuente: elaboración propia, 2021

Con el propósito de analizar la opinión del estudiantado respecto al manejo de contenidos del profesorado y evidenciar eventuales diferencias significativas a partir de las áreas de conocimiento, se elaboraron tablas de contingencia y se realizó la prueba de contraste de variables Chi-cuadrado $\left(\mathrm{X}^{2}\right)$ para contrastar la hipótesis de independencia entre ambas variables. Como se observa en la tabla 4, se obtuvieron índices de relación significativa entre las áreas de conocimiento y las variables "relación con otras materias" y "fomento de la participación": en relación con la frecuencia con que el cuerpo docente relaciona la enseñanza de los contenidos de sus materias con otras materias del plan de estudio, la opinión del estudiantado del área de ciencias sociales (89.9\%) contrasta significativamente con la del área de ciencias administrativas (82.2\%). Lo mismo se observa en la frecuencia con que el profesorado fomenta la participación del estudiantado en el desarrollo de su clase: el área de ciencias sociales $(87.0 \%)$ contrasta de manera significativa con quienes estudian alguna licenciatura del área de ciencias administrativas (82.8\%). 
Tabla 4.

Tablas de contingencia y chi-cuadrada por áreas de conocimiento con base en el manejo de contenidos por parte del profesorado, FCAYS, UABC (México) 2019-1

\begin{tabular}{|c|c|c|c|c|c|c|c|c|c|c|c|c|c|c|c|c|c|c|c|c|}
\hline & \multicolumn{4}{|c|}{ Explicación } & \multicolumn{4}{|c|}{ Dominio } & \multicolumn{4}{|c|}{ Contextualización } & \multicolumn{4}{|c|}{ Otras materias } & \multicolumn{4}{|c|}{ Participación } \\
\hline & \multicolumn{2}{|c|}{ Bajo } & \multicolumn{2}{|c|}{ Alto } & \multicolumn{2}{|c|}{ Bajo } & \multicolumn{2}{|c|}{ Alto } & \multicolumn{2}{|c|}{ Bajo } & \multicolumn{2}{|c|}{ Alto } & \multicolumn{2}{|c|}{ Bajo } & \multicolumn{2}{|c|}{ Alto } & \multicolumn{2}{|c|}{ Bajo } & \multicolumn{2}{|c|}{ Alto } \\
\hline & $n$ & $\%$ & $n$ & $\%$ & $n$ & $\%$ & $\mathbf{N}$ & $\%$ & $n$ & $\%$ & $n$ & $\%$ & $n$ & $\%$ & $n$ & $\%$ & $n$ & $\%$ & $n$ & $\%$ \\
\hline Ciencias Sociales & 127 & 12.9 & 855 & 87.1 & 104 & 10.6 & 877 & 89.4 & 390 & 40.0 & 586 & 60.0 & 99 & 10.1 & 880 & 89.9 & 127 & 13.0 & 851 & 87.0 \\
\hline $\begin{array}{l}\text { Ciencias } \\
\text { Administrativas }\end{array}$ & 123 & 14.4 & 732 & 85.6 & 119 & 13.9 & 735 & 86.1 & 394 & 46.2 & 458 & 53.8 & 152 & 17.8 & 701 & 82.2 & 147 & 17.2 & 706 & 82.8 \\
\hline Ciencias Jurídicas & 95 & 16.8 & 469 & 83.2 & 89 & 15.8 & 476 & 84.2 & 220 & 39.4 & 339 & 60.6 & 86 & 15.2 & 478 & 84.8 & 91 & 16.2 & 472 & 83.8 \\
\hline $\mathrm{X}^{2}$ & & 4.4 & & & & & & & & 9,5 & & & & 23,2 & & & & 6,8 & & \\
\hline Sig. & & .10 & & & & & & & & .0 & & & & .00 & & & & .03 & 33 & \\
\hline
\end{tabular}

Fuente: elaboración propia, 2021

A su vez, se preguntó al estudiantado acerca de las estrategias que utiliza el profesorado para enseñar los contenidos de sus materias, para lo cual se utilizó una escala nominal categórica de 5 opciones: i) exposición oral, repetición/memorización de los contenidos, sin participación del estudiantado; ii) exposición oral con participación activa del estudiantado (por ejemplo, mediante preguntas); iii) diagnóstico de los conocimientos previos y adecuación de los contenidos; iv) aprendizaje individual con base en las capacidades personales de cada estudiante; y v) aprendizaje grupal, a través de la interacción y realización de trabajos cooperativos o colaborativos. Como se observa en la tabla 5 , a nivel general más de la mitad del estudiantado (54.2\%) aseveró que sus docentes privilegiaban la exposición oral de los contenidos con fomento de la participación activa del estudiantado en sus clases. A su vez, cerca de un cuarto de la muestra de participantes (24.2\%) aseguró que sus docentes se inclinan por el fomento del aprendizaje grupal mediante el trabajo cooperativo y colaborativo en torno a los contenidos que enseñan.

Tabla 5.

Estrategias de enseñanza utilizadas por docentes según opinión de estudiantes, FCAYS, UABC (México), 2019-1

\begin{tabular}{|l|c|c|}
\hline & $\mathbf{N}$ & $\mathbf{\%}$ \\
\hline Exposición contenidos & 210 & 12.6 \\
\hline Exposición participativa & 904 & 54.2 \\
\hline Diagnóstico & 74 & 4.4 \\
\hline Aprendizaje individual & 77 & 4.6 \\
\hline Aprendizaje grupal & 404 & 24.2 \\
\hline \multicolumn{1}{|c|}{ TOTAL } & $\mathbf{1 , 6 6 9}$ & $\mathbf{1 0 0 . 0}$ \\
\hline
\end{tabular}

Fuente: elaboración propia, 2021 
Nuevamente, con el propósito de contrastar la hipótesis en torno a la independencia de las variables estrategias de enseñanza y las áreas de conocimiento, se ejecutó el análisis estadístico inferencial chi-cuadrado $\left(X^{2}\right.$, con un $95 \%$ de confianza), el cual compara las frecuencias observadas y las frecuencias esperadas de ambas. Es decir, las frecuencias que teóricamente deberían encontrarse en caso de que dichas variables fueran independientes. Como se observa en la tabla 6 , la prueba $X^{2}$ tomó un valor de 32,665 y arrojó un nivel de significación de .001. Lo anterior permite inferir que efectivamente las variables estrategias de enseñanza y áreas de conocimiento están relacionadas y rechazar la hipótesis de independencias entre ambas.

Tabla 6.

Chi-cuadrado de estrategias de enseñanza por áreas de conocimiento, FCAYS, UABC (México), 2019-1

\begin{tabular}{|c|c|c|c|c|c|c|c|c|c|c|}
\hline & \multicolumn{2}{|c|}{1} & \multicolumn{2}{|c|}{2} & \multicolumn{2}{|c|}{3} & \multicolumn{2}{|c|}{4} & \multicolumn{2}{|c|}{5} \\
\hline & $\mathrm{n}$ & $\%$ & $\mathrm{n}$ & $\%$ & $n$ & $\%$ & $n$ & $\%$ & $n$ & $\%$ \\
\hline Ciencias Sociales & 83 & 11.3 & 373 & 50.9 & 32 & 4.4 & 32 & 4.4 & 213 & 29.1 \\
\hline Ciencias Administrativas & 68 & 11.6 & 319 & 54.4 & 29 & 4.9 & 34 & 5.8 & 136 & 23.2 \\
\hline Ciencias Jurídicas & 59 & 16.9 & 212 & 60.6 & 13 & 3.7 & 11 & 3.1 & 55 & 15.7 \\
\hline $\mathrm{X}^{2}$ & \multicolumn{10}{|c|}{32,665} \\
\hline Sig. & \multicolumn{10}{|c|}{.001} \\
\hline
\end{tabular}

Fuente: elaboración propia, 2021

Los resultados anteriores se ilustran de mejor manera en la figura 1. Si bien es cierto que en todas las áreas de conocimiento predomina la exposición oral de los contenidos con participación activa del estudiantado, esta tendencia se incrementa significativamente dentro de las Ciencias Jurídicas (60.6\%). Por su parte, con relación al fomento del aprendizaje grupal colaborativo, destaca el predominio del área de Ciencias Sociales, donde el estudiantado asevera mayoritariamente $(29.1 \%)$ que sus docentes utilizan estrategias de enseñanza apuntadas a tal fin. Finalmente, dentro de las estrategias de enseñanza relacionadas con el diagnóstico de los conocimientos previos y con el aprendizaje invididual, con base en las capacidades personales de cada estudiante, no se obervan diferencias significativas entre las áreas de conocimiento, aunque en ambas se presenta un leve predominio de la opinión del estudiantado del área de Ciencias Administrativas. 
Figura 1. Opinión del estudiantado acerca de las estrategias de enseñanza que utilizan sus docentes por áreas de conocimiento

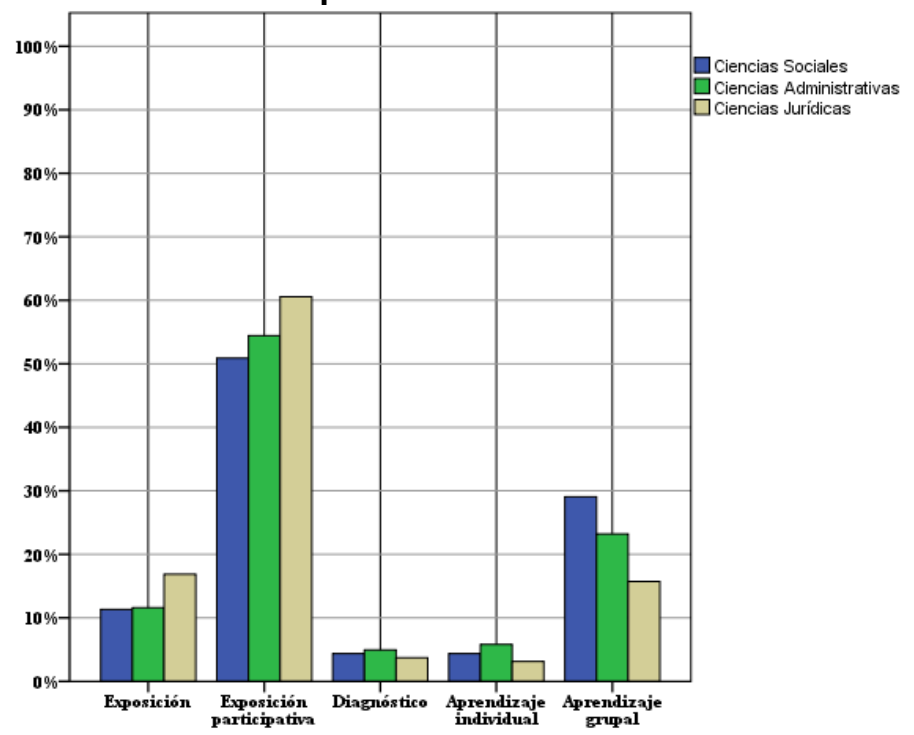

Fuente: elaboración propia, 2021

Relacionado con lo anterior, se indagó acerca de los objetivos de enseñanza que persigue el profesorado según la opinión de sus estudiantes. Para esto, se utilizó una escala nominal categórica de cinco opciones: i) memorizar y reforzar la retención de los contenidos de la materia; ii) asociar los conocimientos previos con los contenidos nuevos de la materia; iii) promover el aprendizaje individual resolviendo situaciones problemáticas según las capacidades del estudiantado; iv) contextualizar el aprendizaje en situaciones reales, relacionadas con el contexto profesional o la comunidad local; y v) promover el aprendizaje grupal colaborativo mediante la interacción. Como se observa en la tabla 7 , a nivel general más de un tercio del estudiantado (36.5\%) aseveraron que el profesorado propone como principal objetivo de enseñanza contextualizar el aprendizaje en situaciones de la vida real, mientras que menos de un quinto (18.8\%) mencionaron que sus docentes asocian sus conocimientos previos con los contenidos nuevos de las materias que imparten.

Tabla 7.

Objetivos de enseñanza según la opinión de estudiantes, FCAYS, UABC, 2019-1

\begin{tabular}{|c|c|c|}
\hline & $\mathrm{n}$ & $\%$ \\
\hline Memorizar y reforzar & 259 & 14.9 \\
\hline Asociar conocimientos & 327 & 18.8 \\
\hline Promover aprendizaje indiv. & 231 & 13.3 \\
\hline Contextualizar aprendizaje & 635 & 36.5 \\
\hline Promover aprendizaje grupal & 289 & 16.6 \\
\hline TOTAL & 1,741 & 100.0 \\
\hline
\end{tabular}

Fuente: elaboración propia, 2021 
De la misma manera, se realizó una prueba de contraste de hipótesis acerca de la independiencia de las variables objetivos de enseñanza y áreas de conocimiento $\left(\mathrm{X}^{2}\right.$, con un $95 \%$ de confianza). Como se observa en la tabla 8 , la prueba $X^{2}$ tomó un valor de 50,856 y arrojó un nivel de significación de .000. Con base en el indicador anterior, se puede asegurar que efectivamente dichas variables están relacionadas y, por lo tanto, se rechaza la hipótesis de independencias entre ambas.

Tabla 8.

Chi-cuadrado de objetivos de enseñanza por áreas de conocimiento, FCAYS, UABC (México), 2019-1

\begin{tabular}{|c|c|c|c|c|c|c|c|c|c|c|}
\hline & \multicolumn{2}{|c|}{1} & \multicolumn{2}{|c|}{2} & \multicolumn{2}{|c|}{3} & \multicolumn{2}{|c|}{4} & \multicolumn{2}{|c|}{5} \\
\hline & $\mathbf{n}$ & $\%$ & $\mathbf{n}$ & $\%$ & $\mathbf{n}$ & $\%$ & $\mathbf{n}$ & $\%$ & $\mathbf{n}$ & $\%$ \\
\hline Ciencias Sociales & 70 & 9.3 & 168 & 22.4 & 79 & 10.5 & 294 & 39.3 & 138 & 18.4 \\
\hline Ciencias Administrativas & 115 & 18.4 & 103 & 16.5 & 94 & 15.0 & 212 & 33.9 & 101 & 16.2 \\
\hline Ciencias Jurídicas & 74 & 20.2 & 56 & 15.3 & 58 & 15.8 & 129 & 31.5 & 50 & 13.6 \\
\hline$\overline{X^{2}}$ & \multicolumn{10}{|c|}{50,856} \\
\hline Sig. & \multicolumn{10}{|c|}{.001} \\
\hline
\end{tabular}

*1=Memorizar y reforzar; 2=Asociar conocimientos previos y nuevos; 3=Promover aprendizaje individual; 4=Contextualizar el aprendizaje en situaciones de la vida real; $5=$ Promover aprendizaje grupal

Fuente: elaboración propia, 2021

Para ilustrar de mejor manera los resultados anteriores, se construyó una gráfica de barras en la que se observan las tendencias de la opinión del estudiantado por área de conocimiento dentro de cada objetivo de enseñanza (figura 2). Al respecto, la opinión del estudiantado de Ciencias Jurídicas destaca el predominio del objetivo relacionado con memorizar y reforzar la retención de contenidos (20.2\%), así como con promover el aprendizaje individual (15.8\%). Lo anterior contrasta significativamente con la opinión del estudiantado de Ciencias Sociales, en la cual predominan tres objetivos de enseñanza: asociar conocimientos previos con conocimientos nuevos (22.4\%), contextualizar el aprendizaje en situaciones reales (39.3\%) y promover el aprendizaje grupal colaborativo (18.4\%). 
Figura 2. Opinión del estudiantado acerca de los objetivoss de enseñanza que utilizan sus docentes por áreas de conocimiento

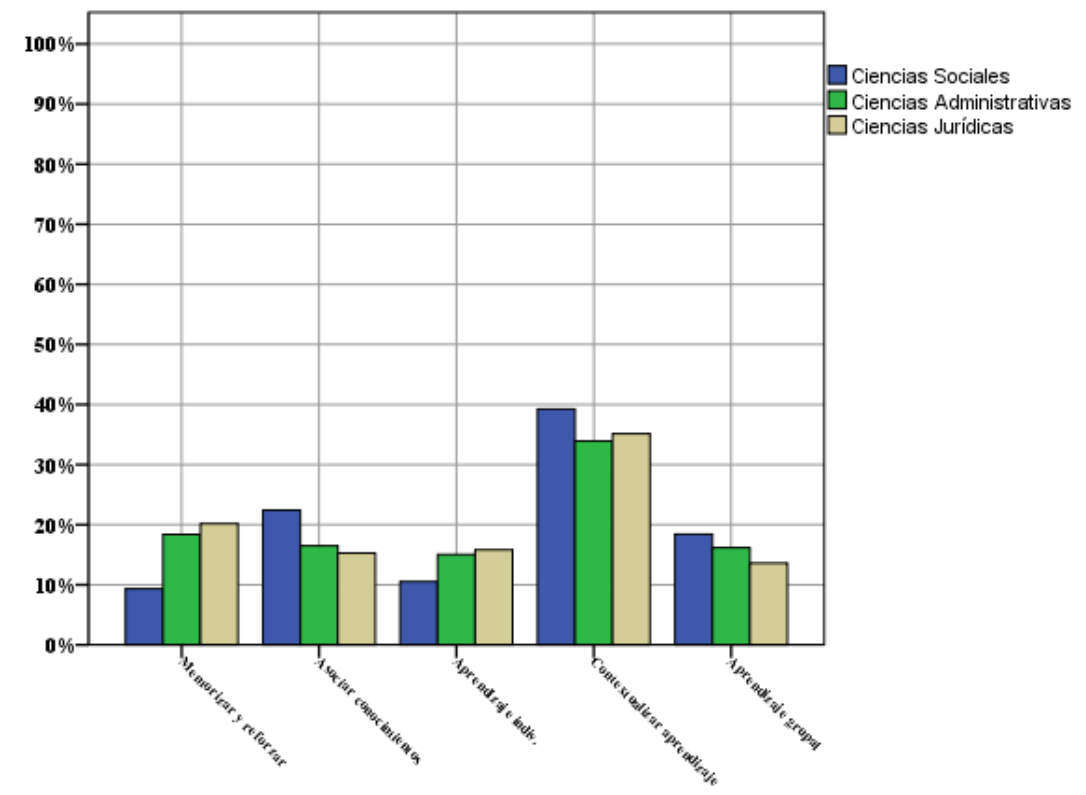

Fuente: elaboración propia, 2021

Por su parte, se indagó la opinión del estudiantado respecto a las estrategias de evaluación del aprendizaje que utilizan sus docentes en torno a los contenidos enseñados. Para esto, se utilizó una escala de Likert de 4 pasos entre el acuerdo y desacuerdo total (codificación 0-3). En la tabla 9 se muestran las ocurrencias y porcentajes reagrupados en dos opciones: desacuerdo y acuerdo. A nivel general, se observan niveles altos con respecto a las estrategias de evaluación del aprendizaje que utilizan sus docentes, por lo que destaca el gusto por estas (84.6\% de acuerdo), la percepción de que a través de dichas estrategias se prioriza el mejoramiento de sus aprendizajes, más allá de la calificación obtenida $(84.3 \%$ de acuerdo) y la inclinación del profesorado por establecer formas de evaluación relacionadas con problemáticas de la vida real ( $83.4 \%$ de acuerdo). Llama la atención que más de la mitad del estudiantado (67.3\%) está de acuerdo en que el profesorado debería incluir estrategias de evaluación del aprendizaje adicionales, mientras que cerca de un cuarto (22.9\%) no cree que aprenda de mejor manera a través de las evaluaciones que aplican sus docentes. 
Tabla 9.

Estrategias de evaluación del aprendizaje según opinión de estudiantes, FCAYS, UABC (México), 2019-1

\begin{tabular}{|l|c|c|c|c|}
\hline \multirow{2}{*}{} & \multicolumn{2}{|c|}{ Desacuerdo } & \multicolumn{2}{c|}{ Acuerdo } \\
\cline { 2 - 5 } & $\mathbf{n}$ & $\mathbf{\%}$ & $\mathbf{n}$ & $\mathbf{\%}$ \\
\hline Me gustan las estrategias de evaluación & 350 & 15.4 & 1926 & 84.6 \\
\hline Aprendo mejor con evaluaciones & 531 & 22.9 & 1788 & 77.1 \\
\hline Retroalimenta posterior a evaluaciones & 466 & 19.9 & 1874 & 80.1 \\
\hline Prioriza mejoramiento de aprendizajes & 366 & 15.7 & 1971 & 84.3 \\
\hline Evaluación auténtica & 386 & 16.6 & 1940 & 83.4 \\
\hline Diferencia distintos ritmos de aprendizaje & 665 & 28.5 & 1671 & 71.5 \\
\hline Debe incluir otras estrategias de evaluación & 765 & 32.7 & 1575 & 67.3 \\
\hline
\end{tabular}

Fuente: elaboración propia, 2021

Por último, para evidenciar eventuales relaciones significativas entre variables asociadas a la opinión del estudiantado respecto a las estrategias de evaluación el aprendizaje utliizadas por sus docentes y las áreas de conocimiento, se realizaron los análisis chi-cuadrado (95\% de confianza) que se muestran en la tabla 10. Cabe destacar que todos los análisis arrojaron índices significativos entre las áreas de conocimiento y la opinión del estudiantado acerca de las estrategias de evaluación utilizadas por sus docentes, excepto la variable que hace referencia a la inclusión de estrategias adicionales. En el resto de la variables relacionadas con esta dimensión, la opinión del estudiantado del área de Ciencias Sociales contrastó de manera significativa respecto a aquellos de Ciencias Jurídicas, quienes se mostraron en desacuerdo en cuanto al gusto por las estrategias de evaluación del aprendizaje utilizadas por sus docentes, a la creencia de que aprenden mejor con dichas estrategias, a la retroalimentación que entregan sus docentes luego de cada evaluación, a la orientación formativa de dichas estrategias apuntadas al mejoramiento de los aprendizajes más allá de la calificación, a la relación que establece el profesorado entre la evaluación del aprendizaje y problemáticas relacionadas con la vida real, así como su preocupación por diferenciar distintos ritmos de aprendizaje en sus estudiantes a través de la evaluaciones que aplica y la adaptación de sus estrategias de enseñanza a dichos ritmos. 
Tabla 10.

Chi-cuadrado de opinión acerca de las estrategias de evaluación del aprendizaje por áreas de conocimiento, FCAYS, UABC (México), 2019-1

\begin{tabular}{|c|c|c|c|c|c|c|c|c|c|}
\hline & \multirow[t]{3}{*}{+2} & \multicolumn{2}{|c|}{ Cs. Sociales } & \multicolumn{2}{|c|}{$\begin{array}{c}\text { Cs. } \\
\text { Administrativas }\end{array}$} & \multicolumn{2}{|c|}{ Cs. Jurídicas } & \multirow{2}{*}{$\mathbf{X}^{2}$} & \multirow{2}{*}{ Sig. } \\
\hline & & $\mathbf{n}$ & $\%$ & $\mathbf{n}$ & $\%$ & $\mathbf{n}$ & $\%$ & & \\
\hline \multirow{2}{*}{$\begin{array}{l}\text { Me gustan las estrategias } \\
\text { de evaluación }\end{array}$} & & 136 & 14.3 & 115 & 14.0 & 99 & 19.6 & \multirow{2}{*}{9,100} & \multirow{2}{*}{.011} \\
\hline & Acuerdo & 812 & 85.7 & 709 & 86.0 & 405 & 80.4 & & \\
\hline \multirow{2}{*}{$\begin{array}{l}\text { Aprendo mejor con } \\
\text { evaluaciones }\end{array}$} & Desacuerdo & 202 & 21.1 & 185 & 22.3 & 144 & 16.5 & \multirow{2}{*}{7,480} & \multirow{2}{*}{.024} \\
\hline & Acuerdo & 757 & 78.9 & 645 & 77.7 & 727 & 83.5 & & \\
\hline \multirow{2}{*}{$\begin{array}{l}\text { Retroalimenta posterior a } \\
\text { evaluaciones }\end{array}$} & Desacuerdo & 163 & 16.9 & 170 & 20.2 & 133 & 24.8 & \multirow{2}{*}{13,299} & \multirow{2}{*}{.001} \\
\hline & Acuerdo & 799 & 83.1 & 671 & 79.8 & 404 & 75.2 & & \\
\hline \multirow{2}{*}{$\begin{array}{l}\text { Prioriza mejoramiento de } \\
\text { aprendizajes }\end{array}$} & Desacuerdo & 133 & 13.9 & 128 & 15.2 & 105 & 19.4 & \multirow{2}{*}{8,133} & \multirow{2}{*}{.017} \\
\hline & Acuerdo & 821 & 86.1 & 715 & 84.8 & 435 & 80.6 & & \\
\hline \multirow{2}{*}{ Evaluación auténtica } & Desacuerdo & 151 & 15.8 & 126 & 15.1 & 109 & 20.3 & \multirow{2}{*}{6,960} & \multirow{2}{*}{.031} \\
\hline & Acuerdo & 803 & 84.2 & 708 & 84.9 & 429 & 79.7 & & \\
\hline \multirow{2}{*}{$\begin{array}{l}\text { Diferencia distintos ritmos } \\
\text { de aprendizaje }\end{array}$} & Desacuerdo & 259 & 26.9 & 229 & 27.4 & 177 & 33.0 & \multirow{2}{*}{7,148} & \multirow{2}{*}{.028} \\
\hline & Acuerdo & 705 & 73.1 & 607 & 72.6 & 359 & 67.0 & & \\
\hline \multirow{2}{*}{$\begin{array}{l}\text { Debe incluir otras } \\
\text { estrategias de evaluación }\end{array}$} & Desacuerdo & 310 & 32.3 & 270 & 32.2 & 185 & 34.2 & \multirow{2}{*}{.726} & \multirow{2}{*}{.696} \\
\hline & Acuerdo & 650 & 67.7 & 569 & 67.8 & 356 & 65.8 & & \\
\hline
\end{tabular}

Fuente: elaboración propia, 2021

\section{Conclusiones}

El presente trabajo de investigación pretende describir la mirada del estudiantado de la FCAYS de la UABC en torno al desempeño docente y trazar algunas inferencias acerca del impacto que poseen las áreas de estudio en las estrategias utlizadas por el profesorado, así como la opinión estudiantil al respecto. En función de lo anterior, se desarrolló esta investigación con base en dos premisas esenciales: por un lado, se plantea que la docencia es una actividad compleja, compuesta de diferentes dimensiones, donde adquiere especial importancia el contexto y las áreas de conocimiento en las que se desarrolla; por otro, que la enseñanza efectiva o lo que significa ser un "buen docente" es un constructo hipotético para el cual no existe una sola forma de evaluación. Al contrario, para poder evaluar de manera integral el desempeño docente se debe recurrir a diferentes agentes educativos (estudiantes, docentes, directores, evaluadores externos) y a distintas estrategias y métodos (coevaluación, autoevaluación, cuestionarios, entrevistas, observaciones de aula) que permitan obtener juicios de valor objetivos y justos respecto a la realidad educativa evaluada. En este sentido, el presente documento aporta elementos para construir una nueva manera de entender el desempeño docente y evaluarlo formativamente según las áreas de conocimiento en la que se desenvuelve el profesorado, trazando un seguimiento procesual de sus estrategias didácticas: las estrategias de enseñanza y las formas de evaluación del aprendizaje pueden 
ser evaluadas como efectivas o buenas dentro de un área, lo cual no se replica en otras cuyos contenidos tienen una naturaleza distinta, como en el caso de las ciencias jurídicas, administrativas y sociales.

En relación con los resultados sobre la dimensión sobre manejo de contenidos, a nivel general se observó que el estudiantado tiene una opinión favorable acerca de la capacidad de explicación y el dominio de los contenidos que enseñan sus docentes. Sin embargo, es importante destacar que cerca de la mitad manifiestó que nunca o solo ocasionalmente sus docentes contextualizan la enseñanza de los contenidos de sus materias con situaciones, experiencias o problemas relacionados con su vida cotidiana. Esto indica que gran parte de sus docentes no están promoviendo procesos de enseñanza-aprendizaje, mediante los cuales se promueve el establecimiento de vínculos significativos entre los contenidos teóricos revisados dentro de las aulas y situaciones, experiencias o escenarios de la vida real, que enfrentan o enfrentarán en su futuro personal y profesional (Díaz Barriga, 2005). De lo anterior, se puede inferir que predominan prácticas docentes eminentemente discursivas, transmisivas y enfocadas en la reproducción de contenidos, en detrimento de prácticas constructivistas apoyadas en la enseñanza focalizada, contenidos adecuados y estrategias de evaluación auténticas, contextualizadas en situaciones problemáticas reales que promuevan el fortalecimiento de los conocimientos procedimentales y actitudinales. Al respecto, más de la mitad del estudiantado opinó que sus docentes privilegian la exposición oral de los contenidos con fomento de la participación activa de estudiantes. Esto indica que el profesorado mantiene un formato de clases tradicional basado en la transmisión oral de los contenidos, aunque promueve la participación activa del estudiantado.

Por otro lado, el hecho de que cerca de un cuarto de la muestra asegurara que sus docentes efectivamente se inclinaban por el fomento del aprendizaje grupal mediante la interacción dialógicsa entre estudiantes en torno a los contenidos educativos, el trabajo cooperativo y la implementación de tareas y trabajos colaborativos, indica que una parte no menos del profesorado se alinea al modelo educativo de la UABC, fundamentado en el humanismo y el constructivismo pedagógico. En este sentido, de acuerdo con Gordon y Debus, (2002) se entiende que el profesorado debe actuar como un mediador capaz de construir un entorno de aprendizaje en que se trabaje para resolver problemas reales, con tareas auténticas, que propicie la indagación, el cuestionamiento y la cooperación entre el estudiantado. 
En cuanto a las estrategias de evaluación del aprendizaje que utilizan sus docentes, se presentan los siguientes resultados. Por una parte, se observan niveles altos de aprobación por parte del estudiantado, el cual valora favorablemente que a través de dichas estrategias se prioriza el mejoramiento de sus aprendizajes. Por otra, llamó la atención que más de la mitad del estudiantado considere que el profesorado debería incluir estrategias de evaluación del aprendizaje adicionales en beneficio de todos, mientras que cerca de un cuarto no cree que aprenda de mejor manera a través de las evaluaciones que aplican sus docentes. Estas tendencias tienes contrastes significativos según él área de estudios del estudiantado, de lo cual se infiere que las prácticas evaluativas de la planta docente varían significativamente entre los docentes que enseñan ciencias sociales, administrativas y jurídicas.

Al respecto, el presente documento propone que a nivel general e independientemente del área de conocimiento, se debe de privilegiar el uso de estrategias de evaluación formativas y continúas durante el desarrollo del proceso de enseñanza-aprendizaje que proporcionen indicios y recomendaciones para la mejora, de tal forma que el estudiantado vaya interiorizando criterios de calidad que le permitirán aprender a autoevaluarse y a mejorar el propio proceso de aprendizaje. En ese sentido, se entiende que las actividades de evaluación del aprendizaje son instancias en las que se pueden promover, enriquecer y continuar los procesos de aprendizaje (Álvarez Méndez, 2001).

Claramente los resultados de este estudio están sesgados dado que se basa en la perspectiva de un solo sujeto educativo (estudiantado) que emite juicios de valor respecto al desempeño de sus docentes a través de un instrumento de respuesta cerrada. Estas limitaciones se podrian superar incluyendo la mirada de otros sujetos educativos y ampliando los horizontes metodológicos a través del uso de otros métodos (cualititavos, mixtos) y otras herramientas (entrevistas en profundidad, grupos de discusión, observación de aula) para la recolección de información en torno a este objeto de estudio.

Por último, al contrastar la opinión del estudiantado respecto al desempeño de sus docentes por área de conocimiento se observaron diferencias significativas que permiten inferir ciertas ideas al respecto. En efecto, la opinión del estudiantado del área de ciencias jurídicas y de ciencias administrativas contrastó significativamente con aquellos de ciencias sociales: respecto al dominio de contenidos, estos muestran diferencias significativas con aquellos de ciencias administrativas cuando les preguntan acerca de la relación que establecen sus docentes entre los contenidos de sus materias con otras del plan de estudio. Asimismo, la opinión de ambos grupos de estudiantes también contrastó significativamente según el 
fomento de la participación en clases. A su vez, el estudiantado del área de ciencias jurídicas opina que las estrategias de enseñanza de sus docentes se inclinan más hacia modelos tradicionales (transmisivos), lo cual contrasta de manera significativa con aquellos de ciencias sociales, los cuales identifican las estrategias y objetivos de enseñanza de sus docentes con teorías constructivistas. Lo anterior deja el camino abierto para seguir explorando las relaciones que se desarrollan entre las áreas de conocimiento y aspectos estratégicos del desempeño docente en el nivel educativo superior, lo cual puede derivar en propuestas de formación docentes para fortalecer aquellos aspectos débiles según la opinión del estudiantado, especialmente respecto a las estrategias de evaluación del aprendizaje utilizadas por el profesorado.

\section{Referencias}

Abreu, Omar., Naranjo, Miguel., Rhea, Bertha. y Gallegos, Mónica. (2016). Modelo didáctico para la Facultad de Ciencias Administrativas y Económicas de la Universidad Técnica del Norte de Ecuador. Formación Universitaria, 9(4), 3-10. https://doi.org/10.4067/s0718$\underline{50062016000400002}$

Álvarez Méndez, Juan Manuel. (2001). Evaluar para conocer, examinar para excluir. Madrid, España: Morata.

Ávila Feites, Amelia., Quintero, Niria. y Hernández, Gladis. (2010). El uso de estrategias docentes para generar conocimientos en estudiantes de educación superior. Omnia, 16 (3), 56-76. Recuperado de https://www.redalyc.org/pdf/737/73716205005.pdf

Bravo Mancero, Patricia. y Varguillas Carmona, Carmen. (2015). Estrategias didácticas para la enseñanza de la asignatura Técnicas de Estudio en la Universidad Nacional de Chimborazo. Sophia, Colección de Filosofía de la Educación, 19, 271-290. doi: https://doi.org/10.17163/soph.n19.2015.13

Coll, César. y Solé, Isabel (2002). Enseñar y aprender en el contexto del aula. En: C. Coll, A. Marchesi \& J. Palacios. (Coords.), Desarrollo Psicológico y Educación 2. Psicología de la Educación Escolar (pp. 357-386). Madrid, España: Alianza Editorial.

Contreras, Adrián. y Díaz Quero, Víctor. (2007). La enseñanza de la ciencia. Laurus, 13(25), 114-145. Recuperado de https://www.redalyc.org/pdf/761/76111479006.pdf

Díaz Barriga, Frida. (2005). Enseñanza situada: Vínculo entre la escuela y la vida. México: McGraw Hill.

Espinoza-Freire, Enrique., Tinoco-Izquierdo, Wilson Eladio. y Sánchez-Barreto, Ximena del Rocío. (2017). Características del docente del siglo XXI. OLIMPIA, Revista de la Facultad de Cultura Física de la Universidad de Granma, 14(43), 39-53. Recuperado de https://dialnet.unirioja.es/servlet/articulo?codigo $=6210816$ 
Espinoza Silva, Francisco. (2009). Métodos y estrategias para la enseñanza-aprendizaje del Derecho. Daena, International Journal of Good Concscience, 4(1), 31-74. Recuperado de http://www.spentamexico.org/v4-n1/4(1)\%2031-74.pdf

Gómez, Luis. y Valdés, María. (2019). La evaluación del desempeño docente en la educación superior. Propósitos y representaciones, 7(2), 479-515. doi: http://dx.doi.org/10.20511/pyr2019.v7n2.255

Gordon, Christopher. y Debus, Ray. (2002). Developing deep learning approaches and personal teaching efficacy within a preservice teaching education context. British Journal of Educational Psychology, 72(4), 483-511. doi: https://doi.org/10.1348/00070990260377488

Hativa, Nira. (2000). Teaching for effective learning in Higher Education. London: Kluwer Academic Publishers.

Hativa, Nira., Barak, Rachel. y Simhi, Etty. (2001). Exemplary University Teachers: Knowledge and Beliefs Regarding Effective Teaching Dimensions and Strategies. Journal of Higher Education, 72(6), 699-729. doi: https://doi.org/10.2307/2672900

Henríquez, Patricio., Arámburo, Vicente. y Dávila, Evangelina. (2017). Percepción de los estudiantes universitarios acerca de las estrategias pedagógicas y de evaluación del aprendizaje utilizadas por sus profesores: el caso de la FCAYS de la UABC. Memorias electrónicas del XIV Congreso Nacional de Investigación Educativa, COMIE. San Luis Posotí, México.

Jornet, Jesús. y Leyva, Yolanda. (2009). Conceptos, metodología y profesionalización en la evaluación educativa. México: INITE.

Ortiz Fajardo, Claudia Helena. (2009). Estrategias didácticas para la enseñanza de las ciencias naturales. Revista Educación y Pensamiento, (16), 63-72.

Santiago-García, Rosana. y Fonseca-García, César Darío. (2016). Ser un buen profesor. Una mirada desde adentro. EDETANIA, (50), 191-208. Recuperado de https://dialnet.unirioja.es/descarga/articulo/6039921.pdf

Schoenfeld, Alan. (1998). Toward a theory of teaching-in-context. Issues in Education, 4(1) 194. doi: https://doi.org/10.1016/s1080-9724(99)80076-7

Stake, Robert. (2008). La ventaja de los criterios, la esencialidad del juicio. Revista Iberoamericana de Evaluación Educativa, 1(3), 19-28. Recuperado de https://revistas.uam.es/riee/article/view/4652

Stake, Robert. y Cisneros-Cohernour, Edith. (2000). Situational evaluation of teaching on campus. New Directions for Teaching and Learning, 2000(83), 51-72. Recuperado de https://onlinelibrary.wiley.com/doi/10.1002/tl.8305

UNESCO. (2006). Evaluación del desempeño y carrera profesional docente. Un estudio comparado entre 50 países de América y Europa. Santiago, Chile: Oficina Regional de Educación para América Latina y el Caribe. 
Revista indizada en
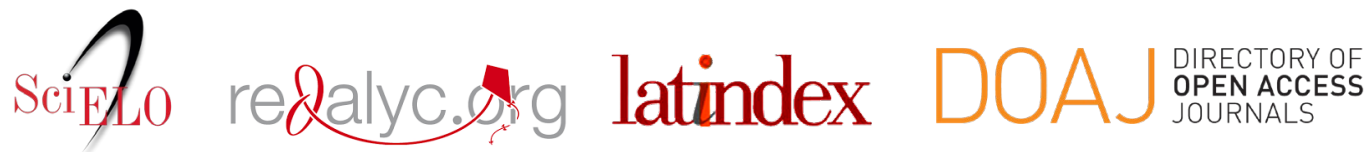

Distribuida en las bases de datos:

- Dialnet

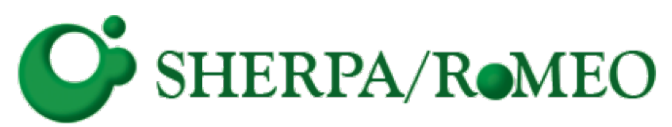

REDIB

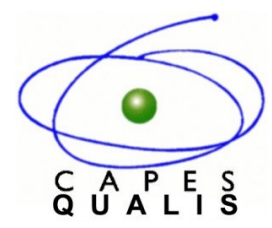

MIAR 